\title{
Virtual Unfolding of the Stomach Based on Volumetric Image Deformation
}

\author{
Kensaku Mori ${ }^{1}$, Hiroki Oka ${ }^{1}$, Takayuki Kitasaka ${ }^{1}$, \\ Yasuhito Suenaga ${ }^{1}$, and Jun-ichiro Toriwaki ${ }^{2}$ \\ 1 Graduate School of Information Science, Nagoya University, \\ Furo-cho, Chikusa-ku, Nagoya 464-8603, Japan \\ kensaku@is.nagoya-u.ac.jp \\ 2 School of Computer and Cognitive Sciences, Chukyo University, Toyota, Japan
}

\begin{abstract}
This paper describes a method for generating unfolded views of the stomach based on 3-D gray image deformation. Unfolded views can show the status of the stomach in one image. The previous method approximates the shape of the stomach using a surface model. This approximation is required for the stretching and reconstruction processes. The previous method had the problem that the intensity information inside the stomach wall was not reconstructed appropriately in the unfolded views, because the stretching process was performed by using only the surface information of the stomach. In the proposed method, we generate the approximated shape using a volumetric model. The volumetric model is deformed so as to generate unfolded views of the stomach. We applied the proposed method to five cases of 3-D abdominal CT images. The experimental results showed that the proposed method can unfolded views of the stomach without any undesirable artifacts. Also, it was possible to visualize the progress of unfolding process.
\end{abstract}

\section{Introduction}

Virtual endoscopy system (VES) is now widely used for visualizing the insides of anatomical structures[1]. The user of the VES can observe the inside of a target organ from arbitrary viewpoints and view directions. The user also can perform fly-through inside the organ by controlling a mouse. Many medical visualization workstations now have the mode of virtual endoscopy. When we examine the insides of organs having large cavities, such as the colon or the stomach, it is possible to diagnose the status of their internal walls by changing the viewpoint and view direction of the VES. However, we have to frequently change the viewpoint and view direction for observing the entire of a target organ's internal wall. This operation requires much time. Frequent change of the viewpoint and view direction sometimes causes oversight regions that are not observed at all during observation. This also leads oversights of important regions such as lesions. If we could generate unfolded views of a target organ, it would be possible to diagnoses the status of the target organ's wall by only one or a few views. The user does not have to change the viewpoint and direction for observing them. 
We have reported a method for generating unfolded views of the stomach 4]. This method virtually stretches the stomach onto a stretching plane and visualizes the unfolded views. We first extract stomach regions from 3-D abdominal CT images. A set of triangles are generated by applying Marching Cubes method to the extracted regions. The shape model, called the approximated shape, which approximates the shape of the stomach, were constricted from the triangles. It consisted of a set of triangles that represent the outer shape of the stomach. The approximated shape was then stretched onto a stretching plane by elastic deformation of the triangles. The deformed image was reconstructed from the input image by using the geometrical relation between the approximated shape and the deformed approximated shape. This method kept geometrical relation only on the triangles. Since the normals of triangles were crossed around the areas that are close to triangle edges, some artifacts were observed in the unfolded views. Also small fold patterns disappeared in the unfolded views.

For solving these problems, this paper shows an improved method for generating unfolded views of the stomach based on volumetric image deformation. The approximated shape is constructed by a set of hexahedrons. These hexahedrons are deformed by using a node and spring model during a stretching process. The deformed image is reconstructed from the relation between the hexahedrons before and after deformation. Unfolded views are obtained by visualizing the deformed images. Since geometric relation is kept in the deformed image by the proposed method, it is possible to reproduce the fold patterns on the stretched views without causing any artifacts.

There are several researches on generating unfolded views of the colon [2] 3]. Most of these methods basically generate unfolded views by: (a) extracting medial axes and (b) generating unfolded views by casting rays from the medial axes. Because simple ray-casting from the medial axes causes tremendous distortion in the generated views, some researchers reported a method for minimizing distortion in the generated views [3. However, the target of these methods is the colon. There is no report about unfolded view generation of the stomach. Also, the above stated method cannot be applied to the stomach, because the stomach has large cavity than the colon and its diameter largely changes along its medial axis. Simple unfolding along the medial axis does not give us satisfactory results in the case of the stomach.

In Sect. 2, we present a method for generating unfolded views based on volumetric image deformation. Sect. 3 shows experimental results of virtual unfolding of the stomach. The unfolding process is also visualized in this section. Brief discussion is given in the same section.

\section{Method}

\section{$2.1 \quad$ Overview}

The proposed method consists of four major steps: (1) generation of an approximated shape, (2) deformation of the approximated shape using the node-spring 


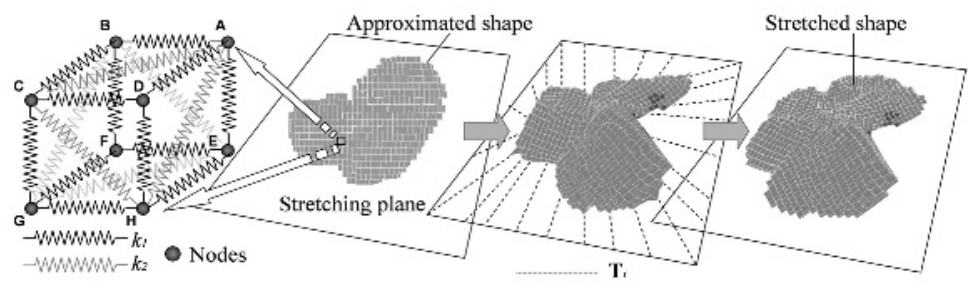

Fig. 1. Elastic modeling and deformation process. (a) Elastic modeling using nodes and springs, (b) unfolding by adding forces that direct to the stretching plane.

model, (3) reconstruction of volumetric image using the relation between the approximated shape and the stretched approximated shape, and (4) visualization of the reconstructed image.

\subsection{Generation of Approximated Shape}

In this step, we model the shape of a target organ by a set of hexahedrons. First we extract the stomach wall by using the method described in 44. We shrink the obtained binary image, which stores the extraction result, by simply sampling the image at every $d$ voxels. For each voxel of the shrunk image, a hexahedron is allocated so that its center coincides with the location of the voxel. The length of each edge of the hexahedron is equal to the sample interval $d$. Two hexahedrons of two adjacent voxels share one surface including four vertices and four edges. The approximated shape is obtained by allocating hexahedrons for all of sample points.

\subsection{Deformation of the Approximated Shape}

This process stretches the approximated shape by using elastic deformation of the hexahedrons. We allocate nodes at the vertices of the hexahedrons. Here we denote eight vertices of one hexahedron as $\mathbf{A}, \mathbf{B}, \mathbf{C}, \mathbf{D}, \mathbf{E}, \mathbf{F}, \mathbf{G}, \mathbf{H}$ (Fig. 1 Springs are allocated on the edges of a hexahedron and on two diagonals on the surface of the hexahedron. Neighboring hexahedrons share nodes and springs. Deformation of the approximated shape is achieved by adding forces, which direct the nodes onto a stretching plane, to the selected nodes. The deformation is computed by iteratively updating the positions of each node based on the force working on each node. The stretching plane is manually allocated by using graphical user interface.

For a hexahedron, we allocate nodes on each vertex and springs on the edges between the vertices AB, BC, CD, DA, AE, BF, CG, DH, EF, FG, GH, HE as illustrated in Fig. 1] The spring constants are set to be $k_{1}$ and the natural lengths are the distances between these vertices. Also we allocate springs in the diagonal direction (between the vertices AC, CH, AH, GE, BE, BG. The 
spring constants of these springs are $k_{2}$. Then we input a cutting line on the approximate shape. The cut of the approximated shape is implemented as cut of springs. Then, we deform the approximated shape by adding forces that direct nodes to a stretching plane. For calculating deformation, we choose tne NodeSpring model rather than the Mass-Spring model for: (a) avoiding undesireble oscillation during the deformation process and (b) finding the equilibrium of the forces.

When the stretching force $\mathbf{F}_{i}(n-1)$ is added to the node $i$, the position of the node $i$ at the $n$-th iteration step is described as

$$
\mathbf{P}_{i}(n)=\mathbf{P}_{i}(n-1)+\rho \mathbf{F}_{i}(n-1),
$$

where $\rho$ is a constant that controls the movement of the node at one iteration step. $\mathbf{F}_{i}(n)$ is force working on the node $i$ and formulated as

$$
\mathbf{F}_{i}(n)=\mathbf{F o}_{i}(n)+\sum_{j \in N_{i}} \mathbf{F n}_{i j}(n),
$$

where $N_{i}$ is a set of nodes connecting to the node $i$. The force $\mathbf{F} \mathbf{n}_{i j}(n)$ is a force caused by the spring between the nodes $i$ and $j$ and represented by

$$
\mathbf{F n}_{i j}(n)=k\left(\left\|\mathbf{R}_{i j}(n)\right\|-l_{i j}\right) \frac{\mathbf{R}_{i j}(n)}{\left\|\mathbf{R}_{i j}(n)\right\|},
$$

where $l_{i j}$ is a natural length of the spring between the nodes $i$ and $j, \mathrm{k}$ a spring constant, and $\mathbf{R}_{i j}(n)$ a vector that directs from the node $i$ to the node $j . \mathbf{F o}_{i}(n)$ is a force for stretching and is working on the nodes that the user specifies. It is formulated as

$$
\mathbf{F o}_{i}(n)=\alpha \mathbf{T}_{i}(n),
$$

where $\mathbf{T}_{i}(n)$ is a vector that directs from the node $i$ to the stretching plane. We add the stretching force until the approximated shape becomes a desired shape.

\subsection{Volumetric Image Deformation}

We deform the input image by using the geometrical relation between the approximated shape and the stretched shape (Fig. 2). All of the hexahedrons of the approximated shape and the stretched shape have one-to-one correspondences. The deformed image is generated by using this relation. We assume that the $i$-th hexahedron $H_{i}$ of the approximated shape corresponds to a hexahedron $H_{i}^{\prime}$ of the approximated shape. We assume that the vertices $\mathbf{A}, \mathbf{B}, \mathbf{C}, \mathbf{D}, \mathbf{E}, \mathbf{F}, \mathbf{G}$, and $\mathbf{H}$ of $H_{i}$ correspond to the vertices $\mathbf{A}^{\prime}, \mathbf{B}^{\prime}, \mathbf{C}^{\prime}, \mathbf{D}^{\prime}, \mathbf{E}^{\prime}, \mathbf{F}^{\prime}, \mathbf{G}^{\prime}$, and $\mathbf{H}^{\prime}$ of $H_{i}^{\prime}$, respectively. When an internal dividing point $\mathbf{P}^{\prime}$ is in the tetrahedron formed by vertices $\mathbf{A}^{\prime} \mathbf{C}^{\prime} \mathbf{D}^{\prime} \mathbf{H}^{\prime}$, the point $\mathbf{P}^{\prime}$ can be represented by

$$
\mathbf{P}^{\prime}=(1-t) \mathbf{A}^{\prime}+t\left(s\left((1-u) \mathbf{C}^{\prime}+u \mathbf{D}^{\prime}\right)+(1-s) \mathbf{H}^{\prime}\right),
$$



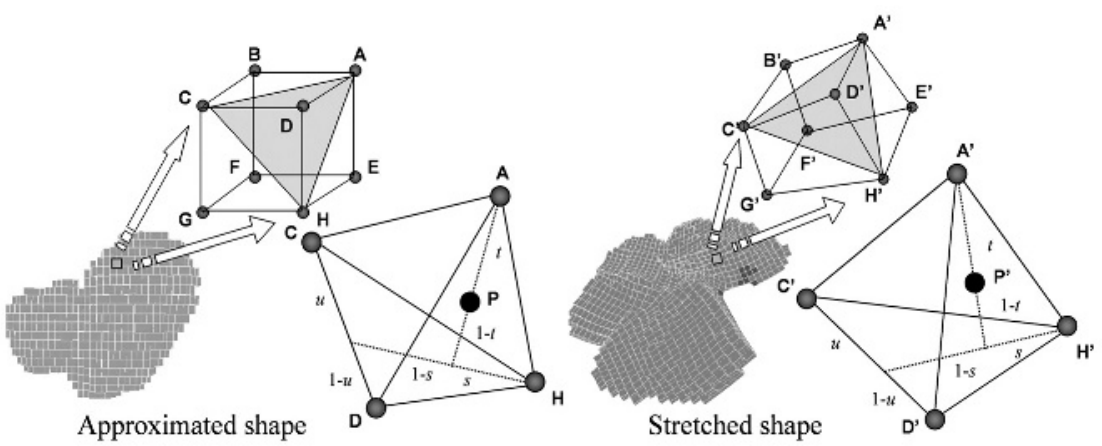

Fig. 2. Geometrical relation between the approximated shape and stretched shape.

where $s, u$, and $t$ are internal dividing parameters and satisfy $0 \leq s, t, u \leq 1$. The point $\mathbf{P}$ corresponding to $\mathbf{P}^{\prime}$ can be expressed as

$$
\mathbf{P}=(1-t) \mathbf{A}+t(s((1-u) \mathbf{C}+u \mathbf{D})+(1-s) \mathbf{H}) .
$$

If the point $\mathbf{P}^{\prime}$ is inside a tetrahedron formed by vertices $\mathbf{A}^{\prime} \mathbf{E}^{\prime} \mathbf{F}^{\prime} \mathbf{H}^{\prime}, \mathbf{A}^{\prime} \mathbf{B}^{\prime} \mathbf{C}^{\prime} \mathbf{F}^{\prime}$, $\mathbf{A}^{\prime} \mathbf{C}^{\prime} \mathbf{F}^{\prime} \mathbf{H}^{\prime}$, or $\mathbf{C}^{\prime} \mathbf{F}^{\prime} \mathbf{G}^{\prime} \mathbf{H}^{\prime}$, the corresponding point $\mathbf{P}$ can be calculated in the same way. The gray value at the point $\mathbf{P}^{\prime}$ in the reconstructed image is computed from the gray values at $\mathbf{P}$ and its neighboring points by linear interpolation. We iterate this process for all points of the reconstruction image.

\subsection{Visualization}

We visualize the reconstructed image where the target organ is stretched by using a volume rendering method. Virtually stretched image are finally obtained from this visualization process.

\section{Experimental Results and Discussion}

We have applied the proposed method to five cases of 3-D abdominal CT images. The parameters are set as $\rho=1, k_{1}=0.5, k_{2}=0.1, \alpha=0.8$. These values are determined experimentally so that the approximated shapes are stretched on the stretching planes satisfactorily for all cases. Sampling interval in the process of the approximated shape generation is set to be $d=8$. It took 60 minutes in the approximated shape generation, 15 minutes for stretching, and 30 minutes in volumetric image reconstruction on a conventional PC (CPU: Intel Pentium III 1.0 GHz, Main memory: $1 \mathrm{~GB}$, OS: Windows 2000). Acquisition parameters of CT images are: $512 \times 512$ pixels, $150-181$ slices, $0.625 \times 0.625 \mathrm{~mm}$ of pixel pitch, $1.0 \mathrm{~mm}$ in slice intervals, and $1.0-5.0 \mathrm{~mm}$ in slice thickness. Figure 3 shows the approximated shape and its unfolded one. The unfolded views obtained by 


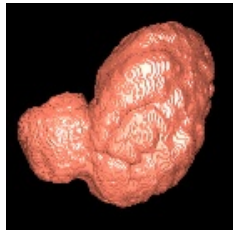

(a)

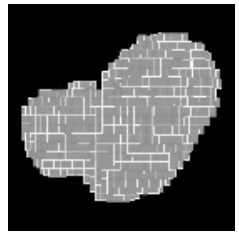

(b)

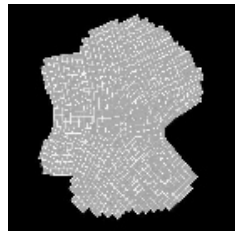

(c)

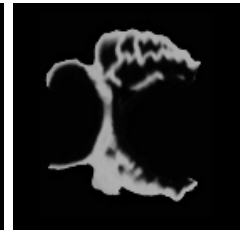

(d)

Fig. 3. Results of unfolding process. (a) Outside view of the stomach, (b) approximated shape, (b) stretched approximated shape, (c) an example of slice images of reconstructed volume.

the previous and the proposed methods are shown in Fig. 4. We visualized the progress of the unfolding process by continuously performing volumetric image reconstruction in Fig. 囵

From the experimental results, it is obvious that the proposed method can generate unfolded views satisfactorily. There are many folds, which have mountain ridge shapes, on the internal wall of the stomach. Because these folds show concentration patterns to lesions in the case of stomach cancer, running directions of folds are very important information for diagnosing the stomach. The unfolded views are quite useful for diagnosing running directions of folds, since it is possible to observe the status of the stomach wall only by one view. Also, the proposed method generates unfolded views basing upon the rule for generating the specimen of a resected stomach defined by Japanese Gastric Cancer Association. Physicians can intuitively understand the status of the stomach by using the generated views.

In the experimental results, the proposed method reproduces small fold patterns very well on the stretched views. Such patterns disappear in the unfolded views obtained by the previous method. Also, some artifacts are observed on the borders of triangles of the approximated shape in the previous method. Such artifacts are not observed on the unfolded views of the proposed method. As illustrated in Fig. 6, although large folds are reproduced well on the unfolded views of the previous method, there are regions that are reproduced with heavy distortion (the part indicated by the arrow in Fig. 6) and some artifacts that do not exist on the original stomach wall. This is because the previous method kept geometric relation only on the triangles. On the other hand, there is no such region in the unfolded views generated by the proposed method. Since the proposed method constructs an elastic model of the stomach wall and then deforms it with keeping its internal geometrical relation by introducing a hexahedron deformation model, few artifacts are observed on the unfolded views.

\section{Conclusions}

This paper presented an improved method for generating stretched views based on volumetric image deformation. We constructed the approximated shape by 


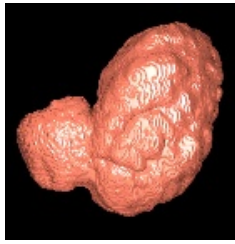

(a)

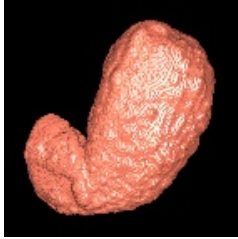

(e)

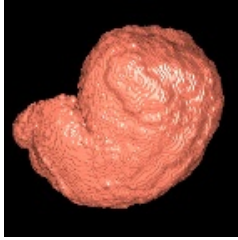

(i)

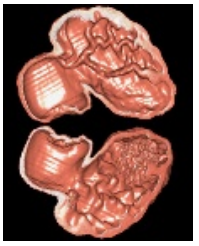

(b)

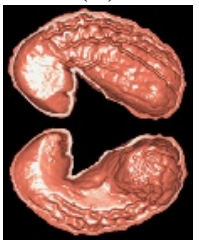

(f)

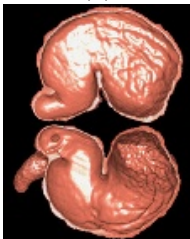

(j)

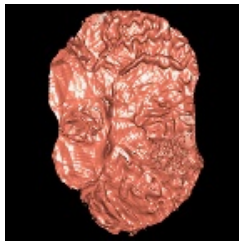

(c)

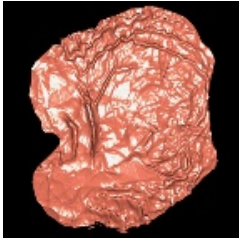

(g)

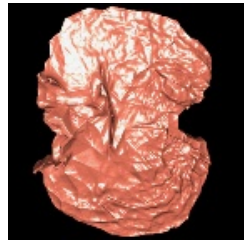

(k)

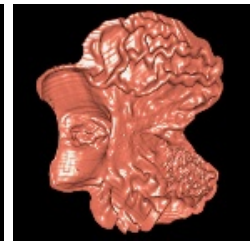

(d)

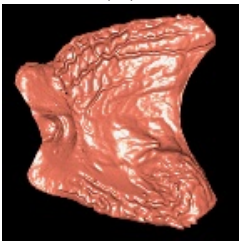

(h)

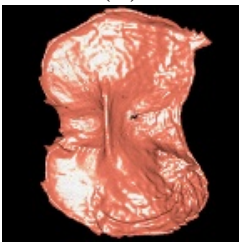

(1)

Fig. 4. Unfolded views. (a,e,i) Outside views, (b,f,j) inside views obtained by dividing stomach regions, $(\mathrm{c}, \mathrm{g}, \mathrm{k})$ unfolded views obtained by the previous method, and $(\mathrm{d}, \mathrm{h}, \mathrm{l})$ unfolded views by the proposed method.

a set of hexahedrons. We deformed these hexahedrons by using a node-spring model and adding stretching forces to them. The deformed image was reconstructed from the relation between the hexahedrons before and after deformation. Since geometric relation was kept in the deformed image by the proposed method, it was possible to reproduce fold patterns on the stretched views without causing any artifacts. The experimental results showed much improvement in the reproduction of fold patterns on the inner wall of the target organ. Future work includes: (a) quantitative analysis of unfolded views, (b) improvement of computation time, and (c) application to many cases.

Acknowledgments. The authors thank to our colleagues for their useful suggestions and discussions. Parts of this research were supported by the 21st century COE program, the Grant-In-Aid for Scientific Research from Ministry of Education, Sports, Science and Technology and the Japan Society for Promotion of Science, and the Grant-In-Aid for Cancer Research from the Ministry of Health and Welfare of Japanese Government. 


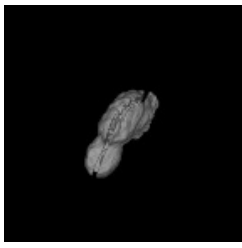

(a)

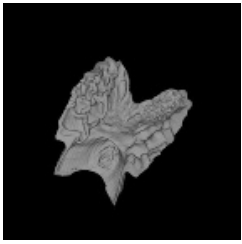

(d)

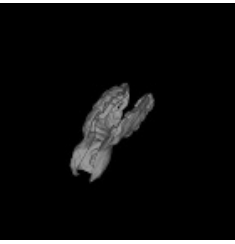

(b)

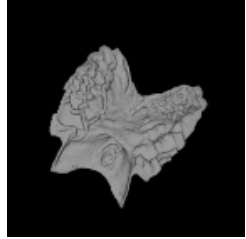

(e)

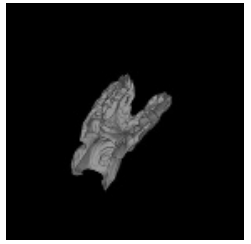

(c)

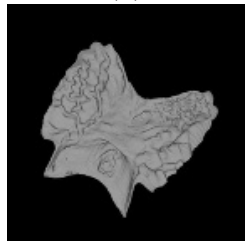

(f)

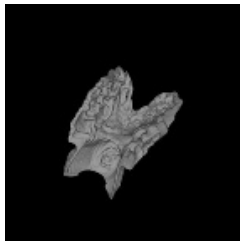

(d)

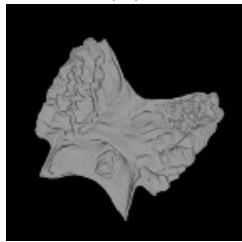

$(\mathrm{g})$

Fig. 5. Visualization results of unfolding process. The unfolding process is performed in time sequence of (a) to $(\mathrm{g})$.

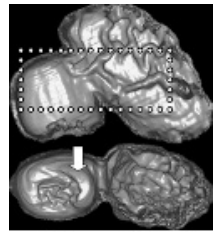

(a)

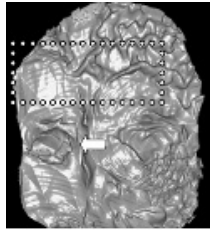

(b)

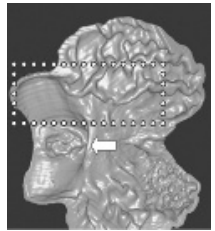

(c)

Fig. 6. Comparison of reproduction results of the fold patterns. The regions indicated by the arrows show distorted region reconstructed by the previous method. There exist artifacts of triangles on the view obtained by the previous method inside the area surrounded by dotted line. (a) Inside view, (b) unfolded view by the previous method, and (c) by the proposed method.

\section{References}

1. P. Rogalla, J. Terwisscha van Scheltinga, B. Hamm, eds., "Virtual endoscopy and related 3D techniques," Springer, Berlin, pp. 946-950, 2001.

2. G. Wang, E. G. McFarland, B. P . Brown et al., "GI Tract Unraveling with Curved Cross Section," IEEE Trans. on Medical Imaging, 17, 2, pp. 318-322, 1998.

3. S. Haker, S. Angenent, A. Tannenbaum, R. Kikinis, "Nondistorting flattening maps and the $3-\mathrm{D}$ visualization of colon CT images," IEEE Trans. on Medical Imaging, 19, 7, pp. 665-670, 2000.

4. H. Oka, Y. Hayashi, K. Mori, et al. "A method for generating unfolded views of organ and its comparison with virtual endoscopy based on undisplayed region rate," Medical Imaging 2003: Physiology and Function: Methods, Systems, and Application, Proceedings of SPIE, vol.5031, pp. 99-101, 2003. 